\title{
Incorporating Sex as a Biological Variable into Clinical and Translational Research Training
}

\author{
Kejal Kantarci, MD, MS, Melissa M. Morrow, $\mathrm{PhD},{ }^{2}$ and Virginia M. Miller, $\mathrm{PhD}^{3}$
}

\begin{abstract}
Incorporating sex as a biological variable (SABV) into basic and medical research requires a deliberate plan that weaves concepts of basic genetics, cellular and molecular physiology, and pharmacology into translational medicine. An R4 approach (Right content to the Right learner at the Right time with the Right modality) allows for content to be available in a variety of formats that reinforces the concepts at staged levels of integration. Weaving SABV throughout the varied formats of the R4 approach within the Clinical and Translational Research Training Programs, into the mentoring and training of scholars in NIH Building Interdisciplinary Careers in Women's Health (BIRCWH), and into the Career Enhancement Core of the Specialized Centers of Research Excellence (SCORE) on sex differences through curriculum, case-based approach and journal clubs, and workshops ensures that learners grasp its fundamental relevancy to their own research and beyond. In addition, the collaborative work among the BIRCWH and SCORE programs brings collective expertise from centers around the United States to individual programs through development of best practices and materials. These collective efforts assure that the next generation of basic, clinical, and translational scientists will bring the dimension of SABV into their research and clinical practice.
\end{abstract}

Keywords: sex differences, training, translational research, sex as a biological variable, SABV

\section{Introduction}

T HE STUDY OF SEX as a biological variable (SABV) is a fundamental concept across all aspects of health and disease and a cornerstone of precision medicine. ${ }^{1}$ However, historically, education focused on women's health, sex, and gender has largely been included within only the reproductiverelated curriculum. To reach the full potential of research and medical advancements, it will be necessary to adopt educational approaches that engage the learner in a curriculum that integrates SABV throughout the full continuum of clinical and translational research training and education. Some approaches will need to be individualized to specialized environments as not all institutions can adopt the recommended integration of SABV across the education continuum due to structural and cultural barriers. Thankfully, there are many existing resources that can be utilized as is or adapted to remove the barrier of developing learning materials de novo. ${ }^{2-5}$

The cornerstone of the clinical and translational science training at the Mayo Clinic is the R4 approach, which promotes continuous development of the workforce that will contribute to the formation of multidisciplinary teams in clinical and translational science. ${ }^{6} \mathrm{R} 4$ approach delivers the Right content to the Right learner at the Right time with the Right modality. A core tenant of this approach is the integration of the training on SABV into research education starting from graduate/medical school and continuing through the clinical training programs that include formal curriculum, case reports/seminars/journal clubs, and workshops. This commentary reports our experience and provides a framework upon which to build an inclusive and wellintegrated SABV concentration throughout clinical and translational research training and education while keeping the learner at the center of the approach.

\section{Curriculum}

SABV can be threaded into the clinical and translational science education using a paradigm for medical education that follows the classic translational research model as proposed by Jenkins and Miller. ${ }^{1}$ This paradigm starts with a discovery phase where the problem is identified and continues with the

Departments of ${ }^{1}$ Radiology, ${ }^{2}$ Health Sciences Research, and ${ }^{3}$ Surgery and Physiology and Biomedical Engineering, Mayo Clinic, Rochester, Minnesota.

(C) Kejal Kantarci et al. 2020; Published by Mary Ann Liebert, Inc. This Open Access article is distributed under the terms of the Creative Commons Attribution Noncommercial License (http://creativecommons.org/licenses/by-nc/4.0/) which permits any noncommercial use, distribution, and reproduction in any medium, provided the original author(s) and the source are cited. 
development of educational methodologies for embedding SABV into education programs. Curricular guidelines are established before the implementation in real-world academic settings. Dissemination to the programs can occur after the implementation is achieved with favorable outcomes.

A curriculum outline of basic topics that may facilitate incorporation of SABV into clinical and translational research are listed in Table 1 that has been developed from our experience and best practices shared by Building Interdisciplinary Careers in Women's Health (BIRCWH) investigators. We are developing videos on these basic content areas, linking to other BIRCWH programs, and have revised the statistics and epidemiology courses in the Center for Clinical and Translational Science to incorporate sex difference analysis.

Despite these efforts, bringing concepts of sex- and gender-based-medicine into the general medical and graduate curriculum continues to be a challenge. Success is limited, in part, by instructors who are not comfortable with the material and a latent concept that a single course on sex differences is the answer. To address these problems, faculty engaged with the BIRCWH and Specialized Centers of Research Excellence (SCORE) programs continue to develop content as part of general seminar programs that have an interdisciplinary reach (e.g., Women's Health Grand Round, Biomedical Engineering Seminars, Center for Individualized Medicine bimonthly program, Center for Diversity and Community Engagement, Cardiovascular Research Seminar Series, Center for Clinical and Translational Science Seminar Series, and MD/PhD education programs).

Collectively, these collaborative programs represent deliberate and targeted approaches to train and disseminate information about women's health and sex-based differences in health and disease to the broader scientific and medical communities, and engage research leaders from multiple disciplines.

In addition, through the BIRCWH and SCORE career enhancement programs, funding is available for pilot studies on sex differences that are meant to attract new investigators into areas of women's health and sex differences research. Recipients of these awards are required to present their findings to various groups, including BIRCWH and SCORE

Table 1. Curriculum Outline of Basic Topics that FACILITATE INCORPORATION OF SEX AND GENDER into Research Design and Analysis

Basic definitions of sex and gender

Review of sex chromosomes: genes, $\mathrm{X}$ inactivation, $\mathrm{X}$ dose effect, sex chromosome effects

Hormonal effects: organization versus activation, cellular signaling, integrative actions, and feedback loops

Interactions: sex by age by hormonal status; sex and hormonal compensation; influence of gender (culture, environment, and psychosocial). What can and cannot be measured. Discussion of health disparities and ethical implications

Pharmacogenomics: genetic variants sex chromosome, enzymes for hormone metabolism, others

Expression of sex differences in health and disease (can be tailored to type and stage of learner; can go by physiological systems or examples for diseases; case studies)

Study design and statistical assessments

Practical examples: articles and grants leadership for guidance and critique, their individual departments and professional societies to disseminate the information more broadly. These small awards are meant to increase the pool of investigators and mentors in sex differences topics who are mentored to become research leaders and mentors. In other words, a three-step "hear one, do one, teach one" approach allows these investigators to (1) learn the concepts, (2) apply them to their own research program, and (3) explain the concepts and provide practical tips on implementation to others with a multiplying effect.

\section{Case-Based Approach and Journal Clubs}

A case-based learning approach may be effective for medical students, residents, and fellows in clinical training programs. In this group, integrating SABV into case discussions and in research discussions such as journal clubs are an effective way of communicating and teaching the value of investigating $\mathrm{SABV}$ in clinical and translational research. An insightful experience is having each learner select an article from their specialty's highest impact journal and evaluate if and how data are presented, and analyzed by sex or gender.

Competencies include (1) being able to define sex and gender, (2) describing the impact of sex and gender on disease mechanisms and progression within the scope of what is currently known, (3) discussing how reporting sex and gender adds to transparency and reproducibility of data, (4) discussion of challenges for experimental design and statistical analysis, and (5) discussion of what future studies are needed to better understand how sex impacts processes associated with mechanisms, progression, and response to treatment to improve individualized patient care. It is expected that having the knowledge base on these topics would ultimately lead to the successful integration of $\mathrm{SABV}$ into the clinical practice and research.

\section{Workshops}

Although the proposed curriculum is an important facilitator for learners, the true test of learning the concepts associated with incorporating SABV into their research is through implementation. Workshops on integrating SABV into a research study design and specific research questions or scenarios may be more appropriate for individuals who are in research careers such as postdoctoral fellows and faculty. During these workshops, SABV is discussed in an interactive environment where the attendees present their specific aims and how they are planning to integrate the SABV into their research plan. In this case, learning is through discussion facilitated by the workshop director and peer-to-peer interaction.

Through collaborations among the BIRCWH and SCORE programs in conjunction with the Mayo Clinic Women's Health Research Center, grant workshops on how to incorporate SAVB into grants have been held three times a year 1 month before NIH grant deadlines. These workshops include a brief didactic on sex differences, the NIH mandate, and suggestions about data presentation and analysis. Attendees are encouraged to bring their long-term goal and specific aims, and then work in groups to reframe these from the standpoint of clinical and experimental evidence about what is known or unknown about how sex and gender may influence the outcome (disease, treatment, or molecular pathway). 
At Mayo Clinic, eight workshops were held between April 2017 and October 2019. These programs are enterprise-wide and include attendees from Mayo Clinic at Rochester, Minnesota, Jacksonville, Florida, and Scottsdale, Arizona, by video conferencing. Attendees $(n=93)$ have included 40 PhDs, 37 MDs, 15 students, and 1 research administrator. A brief survey was sent to these attendees in October 2019; 27 surveys were completed (29\%). Of those completing the survey, all indicated that the content adequately addressed the $\mathrm{NIH}$ requirements for consideration of SABV, and that the material and discussion changed their thinking about the relevance, strategy, experimental design, and statistical analysis used in their grants.

However, only 20 of $26(77 \%)$ attendees who submitted grants reported that they incorporated concepts of SABV into their application. Of those 20, 6 were funded (2 U01, 2 R01, 2 intramural grants). Although there are many reasons why an application may not be successful, all six of the applications that did not consider SABV into their application were not funded. To determine how SABV was reviewed in summary statements, 12 of the $26(46 \%)$ responded that the summary statement found the consideration of SABV to be satisfactory, 1 was found unsatisfactory $(4 \%)$, and there was no comment on SABV in the summary statement for $13(50 \%)$. Based on this survey, we conclude that the grants that get funded always consider SABV in their research plan, but the study section's response is expressed in the summary statement only half of the time.

\section{Conclusions}

$\mathrm{SABV}$ is approached as a fundamental concept for research design. Identifying a basic set of concepts that can be introduced through multiple formats (classroom, selfdirected computer-based programs, and workshops) reinforces the concepts among the learners, and allows them to understand how the fundamental concepts of sex differences apply broadly to research beyond their primary area of interest.

\section{Author Disclosure Statement}

No competing financial interests exist.

\section{Funding Information}

The study was supported by NIH grants HD065987, AG044170, and AG057547.

\section{References}

1. Jenkins MR, Miller VM. 21st century women's health: Refining with precision. Mayo Clin Proc 2016;91:695-700.

2. Miller VM, Kararigas G, Seeland U, et al. Integrating topics of sex and gender into medical curricula-lessons from the international community. Biol Sex Differ 2016;7(Suppl 1): 44.

3. Miller VM, Bahn RS. Mentoring interdisciplinary research teams for the study of sex and gender differences in health and disease. Glob Adv Health Med 2013;2:109-112.

4. Miller VM, Kaplan JR, Schork NJ, et al. Strategies and methods to study sex differences in cardiovascular structure and function: A guide for basic scientists. Biol Sex Differ 2011;2:14.

5. Miller VM, Rocca WA, Faubion SS. Sex differences research, precision medicine, and the future of women's health. J Womens Health (Larchmt) 2015;24:969-971.

6. Weavers K, Mattson DFK, Smith G, Warner D. The R4 Approach to Research Education Program Development: The CTS Experience. Washington, DC: Mayo Clinic and Karolinska Institute Research Conference, 2014.

Address correspondence to:

Kejal Kantarci, MD, MS

Department of Radiology

Mayo Clinic

200 First Street SW

Rochester, MN 55905

E-mail: kantarci.kejal@mayo.edu 